\title{
An Extended Solar Cycle 23 with Deep Minimum Transition to Cycle 24: Assessments and Climatic Ramifications
}

\author{
ERnest M. Agee, Emily Cornett, And Kandace Gleason \\ Department of Earth and Atmospheric Sciences, Purdue University, West Lafayette, Indiana
}

(Manuscript received 6 May 2010, in final form 22 June 2010)

\begin{abstract}
The extended length of solar cycle 23 and the associated deep quiet period (QP) between cycles 23 and 24 have been examined using the international sunspot record from 1755 to 2010 . This study has also introduced a QP definition based on a (beginning and ending) mean monthly threshold value of less than 10 for the sunspot number. Features addressed are the length and intensity of cycle 23, the length of the QP and the associated number of spotless days, and the respective relationships between cycle intensity, length, and QP. The length of cycle 23 (153 months) is second only to cycle 4 (164 months), with an average of 132.5 months for the 11-yr cycle. The length of the QP between cycles 23 and 24 ranks eighth, extending from October 2005 through November 2009 (but subject to continued weakness in cycle 24). The number of spotless days achieved within this QP was 751 (and for all days within the transition from cycle 23 to cycle24, a record number of 801 spotless days had been observed through May 2010). Shortcomings of solar-convection-model predictions of sunspot activity and intensity are also noted, including the failure in the initial predictions of cycle24 onset. The relevance of an extended quiet solar period and the potential reduction of total solar irradiance (TSI) are also discussed, both in the context of climate-model simulations of future climate change as well as with regard to future satellite measurements of TSI.
\end{abstract}

\section{Introduction}

The quasi-periodicity of the 11-yr sunspot cycle has been well documented by the international sunspot number (SSN) record from 1755 to present (the so-called modern era of recording sunspot activity). Prior to this modern period, the telescope was used to observe sunspots (beginning in 1610); however, there was a prominent period of solar inactivity known as the Maunder minimum (1645-1715). Historically, this quiet period (QP) has been attributed to a cold era known as the "Little Ice Age" under the premise that a quiet sun is a cooler sun that is capable of inducing climate change. Today, thanks to instruments placed on satellites outside the earth's atmosphere (see Woods and Lean 2007), the total solar irradiance (TSI) has been measured and thus is known to vary by $1-2 \mathrm{~W} \mathrm{~m}^{-2}$ between maximum and minimum activity, at least for the last three 11 -yr cycles. It is generally understood that the increased luminosity of the strong

Corresponding author address: Ernest M. Agee, Dept. of Earth and Atmospheric Sciences, Purdue University, 550 Stadium Mall Dr., West Lafayette, IN 47907-2051.

E-mail: eagee@purdue.edu solar cycles during the past $100 \mathrm{yr}$ may have contributed to global warming; however, the results have been secondary to contributions by the warming effects of greenhouse gases (GHG) [particularly carbon dioxide $\left(\mathrm{CO}_{2}\right)$ and methane $\left(\mathrm{CH}_{4}\right)$ ], as discussed by Ammann et al. 2007 (also see Solomon et al. 2007). More recently, Fröhlich (2009) has noted that the TSI value for the QP between cycles 23 and 24 was $0.2 \mathrm{~W} \mathrm{~m}^{-2}$ less than the TSI value for the QP between cycles 22 and 23 . This result has potential longterm implications for the role of solar variability in climate change (in particular, global cooling, given an extended grand minimum in solar activity). This will be discussed in more detail in a later section.

In recent years the prediction of the 11-yr sunspot cycle has gained in popularity, based largely on the solar dynamo model [see Dikpati et al. (2006) as well as the review by Woods and Lean (2007)]. Figure 1 shows the respective initial predictions of cycle 24 by the National Center for Atmospheric Research (NCAR) and the National Aeronautics and Space Administration (NASA) models, which targeted cycle 24 to begin in late 2006, achieving continued noteworthy maximum strength by 2011 (comparable to that of recent successive strong cycles). The extended lapse of time between the end of cycle 23 and the start of 


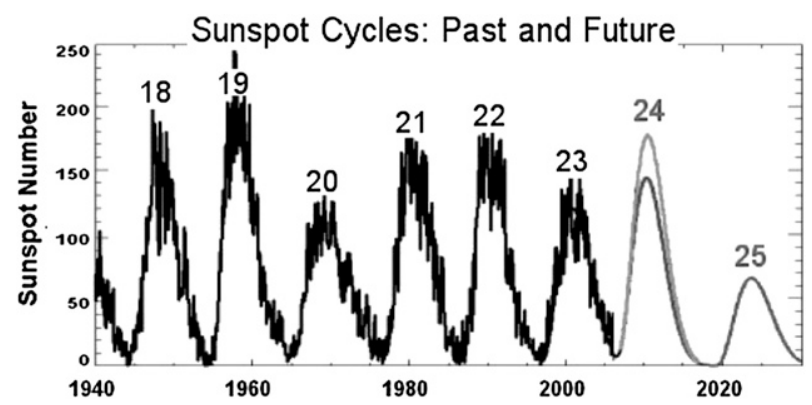

FIG. 1. Hathaway's initial prediction (lower curve) for cycles 24 and 25, and NCAR's prediction for cycle 24 (see Dikpati et al. 2006; Hathaway 2006).

cycle 24 raises the interesting question, Is the extended length of solar cycle 23 unusual? Figure 2 shows the monthly sunspot number from June 1996 to March 2010, which illustrates the extended length of cycle 23 as well as the "deep" QP. Also depicted is the late start of cycle 24 and an illustration of the August 2009 predicted SSN of 40 versus the observed value of 0 (all days in August were spotless).

\section{Solar cycles: Transition, cycle length, and QP}

The determination of transition between cycles is sometimes difficult to assess, and it may not be widely known until solar activity has moved well into the next cycle. Several factors, however, can be considered in identifying the transition, such as 1) observation of the first sunspot of reversed polarity, 2) observation of the first full day with no spots, and 3) an equal count of spots with opposing polarity (the choice for defining cycle transition in this study). The period for an adequate count of polarity spots, however, is sometimes extended because of the lack of sunspot activity. Concern about the extended length of cycle 23 began to appear when a news release by the National Oceanic and Atmospheric Administration (NOAA) on 25 April 2007 stated that the next 11-yr cycle would start in March 2008 (1 yr later than originally expected) and peak in late 2011 to mid-2012. Continued delay in the start of cycle 24 became increasingly noteworthy, highlighted by weak or no daily sunspots at most observatories for the entire month of August 2008 (the first month of zero activity for any single observatory since 1913). E-mail exchanges with J. Lean at the Naval Research Laboratory in the autumn of 2008 suggested that two additional years of solar inactivity would be required to upset the current thinking about the 11-yr sunspot cycle. The absence of solar activity continued, with 71 consecutive days with weak or no daily sunspots, from 10 July through 20 September 2009. Even though an occasional reversed-polarity sunspot was observed, the extended QP between cycles 23 and 24 heightened the interest of the solar physics community. NASA issued a request for proposals (available online at http://nspires.nasaprs.com/) entitled, "Causes and Consequences of the Minimum of Solar Cycle 23" to request research proposals to study this extended QP. It was also noted by J. Janssens of the Belgian Astronomical Association's Solar Section that the

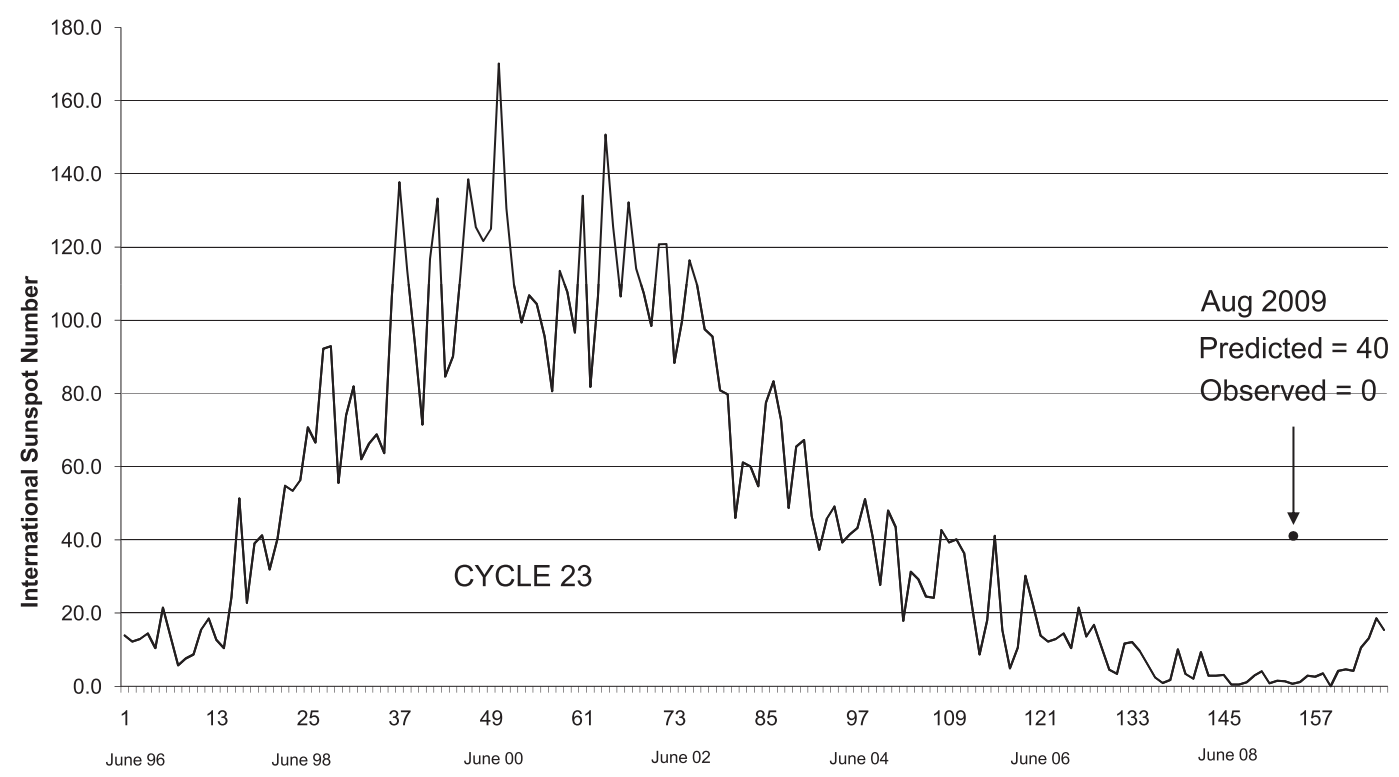

FIG. 2. Plot of monthly values of international SSN for cycle 23 and start of cycle 24 (June 1996-March 2010). Also shown is the August 2009 SSN prediction, as initially provided by NOAA, NCAR, and NASA model forecasts. 
TABLE 1. Observed periods of 11-yr sunspot cycle. Data were provided by the Solar Influences Data Analysis Center in Belgium and the NOAA Space Weather Prediction Center.

\begin{tabular}{cllr}
\hline \hline Cycle No. & \multicolumn{1}{c}{ Start date } & \multicolumn{1}{c}{ End date } & No. years \\
\hline 1 & March 1755 & June 1766 & 11.25 \\
2 & June 1766 & June 1775 & 9.00 \\
3 & June 1775 & September 1784 & 9.25 \\
4 & September 1784 & May 1798 & 13.67 \\
5 & May 1798 & December 1810 & 12.58 \\
6 & December 1810 & May 1823 & 12.42 \\
7 & May 1823 & November 1833 & 10.50 \\
8 & November 1833 & July 1843 & 9.75 \\
9 & July 1843 & December 1855 & 12.42 \\
10 & December 1855 & March 1867 & 11.25 \\
11 & March 1867 & December 1878 & 11.75 \\
12 & December 1878 & March 1890 & 11.25 \\
13 & March 1890 & February 1902 & 11.92 \\
14 & February 1902 & August 1913 & 11.50 \\
15 & August 1913 & August 1923 & 10.00 \\
16 & August 1923 & September 1933 & 10.08 \\
17 & September 1933 & February 1944 & 10.42 \\
18 & February 1944 & April 1954 & 10.17 \\
19 & April 1954 & October 1964 & 10.50 \\
20 & October 1964 & June 1976 & 11.67 \\
21 & June 1976 & September 1986 & 10.25 \\
22 & September 1986 & May 1996 & 9.67 \\
23 & May 1996 & November 2009 & 13.58 \\
24 & December 2009 & (To be determined) & \\
\hline & & &
\end{tabular}

cumulative number of spotless months since the first spotless day on 27 January 2004 is rather large, and comparable to the older cycles (11-14 observed in the nineteenth century), and maybe as long as cycle 4 (see online at http://users.telenet.be/j.janssens/Spotless/Spotless.html\# Period). Again, referring back to Fig. 2, it is evident that cycle 24 has moved out of the deep minimum between cycles 23 and 24 and that, further, all subsequent spots (although weak) now show new cycle polarity. It is also noteworthy that there has been a record number of 801 spotless days in the transition from cycle 23 to cycle 24 (through May 2010), which can be compared with an average of 485 spotless days for all 23 cycle transitions.

\section{a. The frequency of the 11-yr cycle (cycles 1-23)}

Table 1 shows the frequency interval of the past twentythree 11-yr cycles, from March 1755 through November 2009 (the end of cycle 23), which varies from $9.0 \mathrm{yr}$ for cycle 2 to $13.67 \mathrm{yr}$ for cycle 4 . Cycle 23 is of nearly record length at $13.58 \mathrm{yr}$.

A graphical plot provided in Fig. 3 further depicts that cycle 23 is an extreme event but is not outside the extreme limit of past observations. It is further noted in Fig. 3 that there appears to be a cyclical nature to the periodicity of the 11-yr cycle, although it is not statistically significant for the few oscillations shown. As cycle 24 slowly ramps

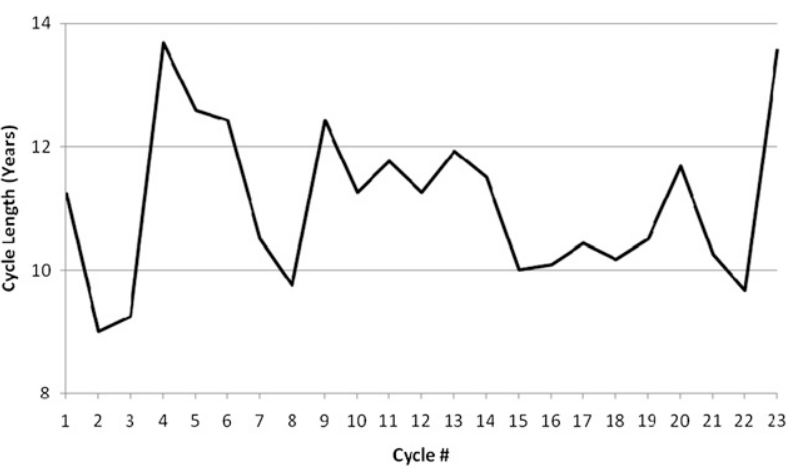

FIG. 3. Length of "11 yr" sunspot cycle from cycle 1 to cycle 23 (March 1755-November 2009).

up [as was expected; see Keating (2008)], revised SSN predictions by the NASA and NOAA models now show a much weaker new cycle. Such weak activity could be a possible reflection of the end to a much longer cycle known as the Gleissberg cycle (the envelope of maxima for eight successive 11-yr cycles).

\section{b. The QP for 11-yr-cycle transition}

A QP has been defined in this study as the period from the first monthly SSN average of $<10$ to the last monthly SSN average of $<10$. The length of the QP during the 23 recorded solar cycles ranges from 6 months for the transition from cycle 1 to cycle 2 to 98 months for the transition from cycle 5 to cycle 6 , averaging to about 37 months overall. At 49 months long, the QP for cycle $23-24$ is longer than this average, but it is actually only the eighth longest and is not particularly extreme. Interestingly, cycle 24 continues (with $100 \%$ reversed-polarity spots); however, the "preliminary" SSN for April 2010 and May 2010 are 7.9 and 8.8 , respectively. This may be consistent with the weakness now predicted for cycle 24 . The reader is also referred to White et al. (2010), which addresses a possible miniature Maunder minimum, with sunspot production ceasing completely by 2015 .

\section{c. Correlations: Cycle intensity, cycle length, and QP length}

Correlations have been made between cycle intensity and duration of the QP (see Fig. 4) and between the length of the solar cycle and thelength of the QP (see Fig. 5). As expected, an inverse correlation (coefficient $R=-0.534$ ) exists between cycle intensity and duration of the QP and a positive correlation $(R=0.664)$ exists between the length of the solar cycle and the length of the QP, although only about $25 \%$ and $50 \%$ of the variance is explained, respectively. Realignment of the plots, to account for phase-shift differences, could yield improved correlations. 


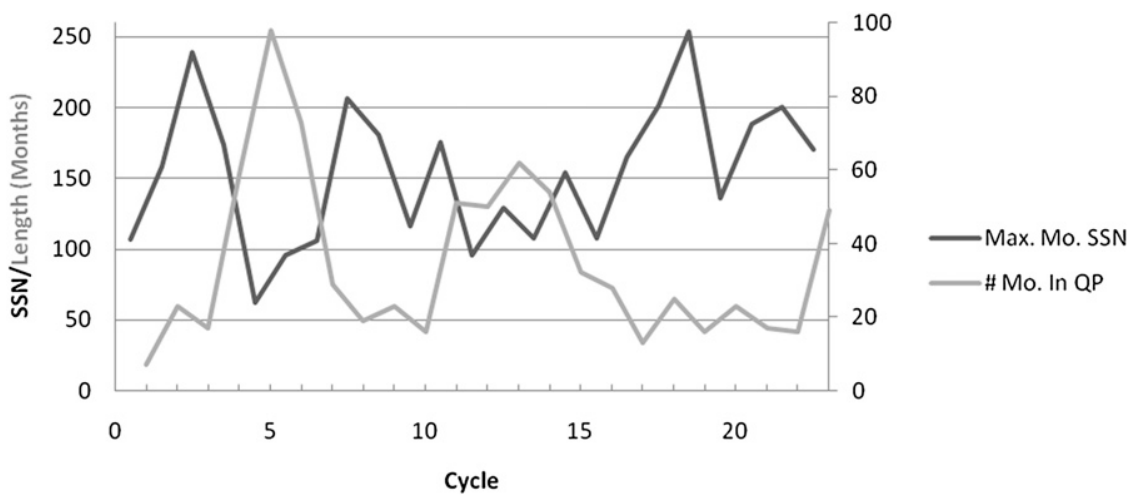

FIG. 4. Depiction of an inverse relationship $(R=-0.534)$ between cycle intensity and duration of the QP.

\section{Solar variability and future climate implications}

As noted in the introduction, the TSI is not a constant, as satellite instruments have clearly documented for three successive 11-yr cycles. Strong solar cycles for the past $100 \mathrm{yr}$ have contributed to global warming, but they are now relegated to a minor role because of the much greater warming effects of GHG forcing (see Scafetta and West 2006a,b). The TSI values from the Total Irradiance Monitor instrument on the Solar Radiation and Climate Experiment (SORCE) satellite showed a TSI minimum during the QP between cycles 23 and 24 of $1361 \mathrm{~W} \mathrm{~m}^{-2}$ in January 2009, which by April 2010 had increased to $1361.4 \mathrm{~W} \mathrm{~m}^{-2}$ with cycle-24 activity. Although Fröhlich (2009) reported QP 23-24 to be $0.2 \mathrm{~W} \mathrm{~m}^{-2}$ less than QP 22-23, this small amount of cooling pales in comparison with $\mathrm{GHG}$ forcing by $\mathrm{CO}_{2}\left(1.66 \mathrm{~W} \mathrm{~m}^{-2}\right)$ and $\mathrm{CH}_{4}$ $\left(0.48 \mathrm{~W} \mathrm{~m}^{-2}\right)$, per Solomon et al. (2007). Please note again that the TSI decreases by about $2.0 \mathrm{~W} \mathrm{~m}^{-2}$ from $11-\mathrm{yr}$ solar maximum to solar minimum. Interestingly, Feulner and Rahmstorf (2010) have reported that even if another Maunder type of grand minimum should occur in the twenty-first century, any contribution to cooling would be minimal and not interruptive to the GHG warming.
Their results were obtained using a coupled climate model with a $0.25 \%$ reduction in TSI, yielding a contribution to cooling of $-0.3^{\circ} \mathrm{C}$ by the year 2100 . The authors appreciate these results but are concerned about certain aspects of the model assumptions and capability. For example, the use by Feulner and Rahmstorf (2010) of model results by Wang et al. (2005) can be called into question when the TSI decrease is so small for a centurylong new grand minimum. Specifically, to assume that $\kappa$ (the diffusion coefficient in nonstationary supergranulation convection) is a constant value of $500 \mathrm{~km}^{2} \mathrm{~s}^{-1}$ should be an assumption of concern. Another concern pertains to reduced TSI during a solar QP, which is actually much less in the UV portion of the solar spectrum, and the Feulner and Rahmstorf model cannot treat the internal model feedback effects for this type of change (G. Feulner 2010, personal communication). Any long-term effect of reduced UV, however, may not be present (see Fröhlich 2009).

In view of the recent unexpected QP behavior by the sun, it becomes increasingly important to continue precise measurements of TSI from satellite platforms. There are no assurances that a new grand minimum could not occur during the twenty-first century, and direct measurement

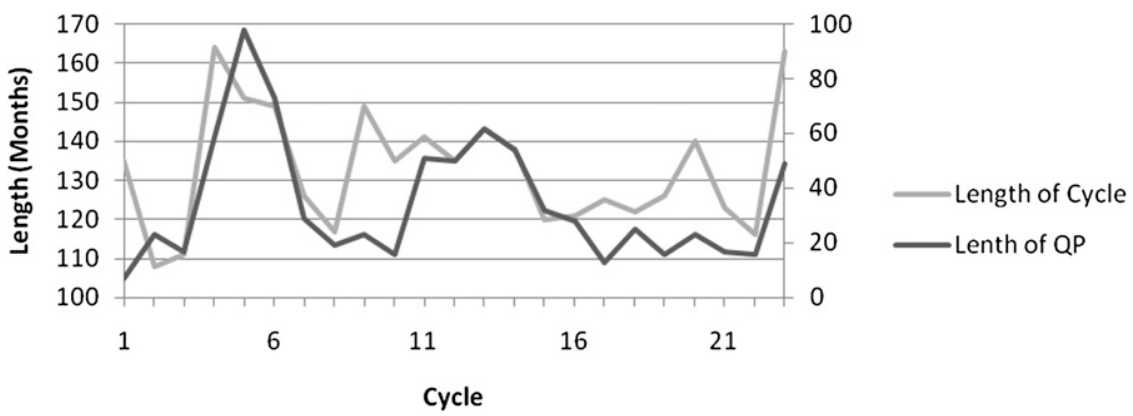

FIG. 5. Depiction of positive correlation $(R=0.664)$ for length of solar cycle vs length of the QP. 
of TSI is the answer needed to guide inferences from proxy data (using cosmogenic radionuclides) or solarmagnetic-model predictions of TSI. Further support of the possibility of continued decrease in solar activity in the twenty-first century is given in a study by Livingston and Penn (2009). Their work shows continuously decreasing magnetic field strength from 1992 to 2009 as well as less vigor in magnetic strength and area of sunspots. Solar observatory and telescope observations (using infrared diagnostics) are equally important as satellite measurements are, and both need to be continued diligently through the twenty-first century of expected climatic change.

\section{Summary}

This study has brought into focus several key aspects of recent solar variability and its place in the sunspot record. Cycle 23 is the second longest on record, and the deep QP between cycles 23 and 24 has set a record for the number of spotless days. Further, this study has introduced a definition for QP transition, which previously has not been well defined (see Henson 2010). The QP between cycles 23 and 24 ranks as the eighth longest in the twentythree 11-yr-cycle records considered from 1755 to 2010, subject to weak cycle-24 activity with preliminary SSN values of 7.9 and 8.8 for April and May 2010, respectively.

This paper also reveals the shortcomings in model predictions of sunspot cycles, which suggest an overall inadequate understanding of the solar dynamo. Studies cited in this paper give adequate cause for future concern about long periods of solar inactivity, comparable to a Maunder type of new grand minimum. Climate models may need to reconsider the importance of reduced TSI for an extended quiet period, given that solar variability (at this point in time) has been relegated to having virtually no effect on predictions of twenty-first-century global warming.

\section{REFERENCES}

Ammann, C. M., F. Joos, D. S. Schimel, B. L. Otto-Bliesner, and R. A. Tomas, 2007: Solar influence on climate during the past millennium: Results from transient simulations with the NCAR Climate System Model. Proc. Natl. Acad. Sci. USA, 104, 3713-3718.

Dikpati, M., G. de Toma, and P. A. Gilman, 2006: Predicting the strength of solar cycle 24 using a flux-transport dynamo-based tool. Geophys. Res. Lett., 33, L05102, doi:10.1029/ 2005GL025221.

Feulner, G., and S. Rahmstorf, 2010: On the effect of a new grand minimum of solar activity on the future climate on earth. Geophys. Res. Lett., 37, L05707, doi:10.1029/ 2010GL042710.

Fröhlich, C., 2009: Evidence of a long-term trend in solar irradiance. Astron. Astrophys., 501, L27-L30, doi:10.1051/0004-6361/ 200912318.

Hathaway, D., cited 2006: Long range solar forecast. [Available online at http://science.nasa.gov/science-news/science-at-nasa/ 2006/10may_longrange/.]

Henson, R., 2010: Looking within the solar minimum. UCAR Magazine, Winter ed., 6-8. [Available online at http://www2. ucar.edu/magazine/features/looking-within-min.]

Keating, C. F., 2008: Not to worry: Solar magnetic activity for cycle 24 is increasing. Eos, Trans. Amer. Geophys. Union, 89, doi:10.1029/2008EO430002.

Livingston, W., and M. Penn, 2009: Are sunspots different during this solar minimum? Eos, Trans. Amer. Geophys. Union, 90, 257-264, doi:10.1029/2009EO300001.

Scafetta, N., and B. J. West, 2006a: Phenomenological solar contribution to the 1900-2000 global surface warming. Geophys. Res. Lett., 33, L05708, doi:10.1029/2005GL025539.

_ , and — 2006b: Phenomenological solar signatures in 400 years of reconstructed Northern Hemisphere temperature record. Geophys. Res. Lett., 33, L17718, doi:10.1029/ 2006 GL027142.

Solomon, S., D. Qin, M. Manning, M. Marquis, K. Averyt, M. M. B. Tignor, H. L. Miller Jr., and Z. Chen, Eds., 2007: Climate Change 2007: The Physical Science Basis. Cambridge University Press, 996 pp.

Wang, Y.-M., J. L. Lean, and N. R. Sheeley Jr., 2005: Modeling the sun's magnetic field and irradiance since 1713. Astrophys. J., 625, 522-538.

White, O. R., W. Livingston, and M. Penn, 2010: Mini Maunder minimum. Extended Abstracts, SORCE Science Meeting, Keystone, CO, Laboratory for Atmospheric and Space Physics, 6B. [Available online at http://lasp.colorado.edu/sorce/ news/2010ScienceMeeting/doc/Session6/6b_White_Livingston_ i.pdf.]

Woods, T. N., and J. Lean, 2007: Anticipating the next decade of the sun-Earth system variations. Eos, Trans. Amer. Geophys. Union, 88, doi:10.1029/2007EO440001. 